\title{
The rotation-lithium depletion correlation in the $\beta$ Pictoris association and the LDB age determination
}

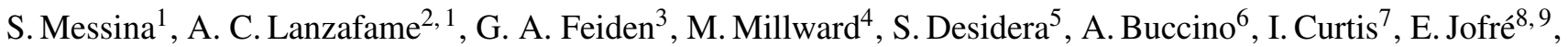 \\ P. Kehusmaa ${ }^{10}$, B. J. Medhi ${ }^{11}$, B. Monard ${ }^{12}$, and R. Petrucci ${ }^{8,9}$ \\ 1 INAF-Catania Astrophysical Observatory, via S.Sofia, 7895123 Catania, Italy \\ e-mail: sergio.messina@oact.inaf.it \\ 2 Universitá di Catania, Dipartimento di Fisica e Astronomia, Sezione Astrofisica, via S. Sofia 78, 95123 Catania, Italy \\ Department of Physics \& Astronomy, Uppsala University, Box 516, 75120 Uppsala, Sweden \\ York Creek Observatory, Georgetown, Tasmania, Australia \\ 5 INAF-Osservatorio Astronomico di Padova, Vicolo dell'Osservatorio 5, 35122 Padova, Italy \\ ${ }^{6}$ Instituto de Astronomía y Física del Espacio (IAFE-CONICET), Buenos Aires, Argentina \\ 7 IAU Minor Planet Center code D79, 2 Yandra Street, Vale Park, 5081 South Australia, Australia \\ 8 Observatorio Astronómico de Córdoba, Laprida 854, X5000BGR, Córdoba, Argentina \\ 9 Consejo Nacional de Investigaciones Científicas y Técnicas (CONICET), 1418 Córdoba, Argentina \\ ${ }_{10}$ Harlingten Atacama Observatory, San Pedro de Atacama, Chile \\ 11 Aryabhatta Research Institute of Observational Sciences, Manora Peak, 263129 Nainital, India \\ 12 Klein Karoo Observatory, Western Cape, South Africa
}

Received 16 March 2016 / Accepted 24 July 2016

\section{ABSTRACT}

\begin{abstract}
Context. Evidence exists in the 125-Myr Pleiades cluster, and more recently in the 5-Myr NGC 2264 cluster, to show that rotation plays a key role in lithium ( $\mathrm{Li}$ ) depletion processes among low-mass stars. Fast rotators appear to be less Li-depleted than equal-mass slow rotators.

Aims. We intend to explore the existence of a Li depletion-rotation connection among the $\beta$ Pictoris members at an age of about $24 \mathrm{Myr}$, and to use this correlation either to confirm or to improve age estimates based on the lithium depletion boundary (LDB) modeling.

Methods. We photometrically monitored all the known members of the $\beta$ Pictoris association with at least one lithium equivalent width (Li EW) measurement from the literature.

Results. We measured the rotation periods of 30 members for the first time and retrieved the rotation periods for another 36 members from the literature, building a catalogue of 66 members with a measured rotation period and Li EW.

Conclusions. We find that in the $0.3<M<0.8 M_{\odot}$ range, there is a strong correlation between rotation and Li EW. For higher mass stars, no significant correlation is found. For very low-mass stars in the $\mathrm{Li}$ depletion onset, at about $0.1 M_{\odot}$, there are too few data to infer a significant correlation. The observed Li EWs are compared with those predicted by the Dartmouth stellar evolutionary models that incorporate the effects of magnetic fields. After decorrelating the Li EW from the rotation period, we find that the hot side of the LDB is well fitted by Li EW values that correspond to an age of $25 \pm 3 \mathrm{Myr}$, which is in good agreement with independent estimates from the literature.
\end{abstract}

Key words. stars: late-type - stars: rotation - starspots - stars: individual: beta Pictoris association - stars: activity - stars: abundances

\section{Introduction}

Recently, much attention has been paid to the $24 \pm 3 \mathrm{Myr}$ (Bell et al. 2015) young $\beta$ Pictoris stellar association. Several studies have enabled the number of confirmed members to increase significantly, leading to the discovery of many more candidate members. Recent works include: Lépine \& Simon (2009); Kiss et al. (2011); Schlieder et al. (2010, 2012); Shkolnik et al. (2012); Malo et al. (2013, 2014a,b). The first comprehensive search for the rotation periods of the low-mass members of $\beta$ Pictoris was carried out by Messina et al. $(2010,2011)$ who retrieved a total of 38 low-mass members (i.e., spectral types from late $\mathrm{F}$ to $\mathrm{M}$ ) from the earlier compilations of Zuckerman \& Song (2004), Torres et al. (2006), and Kiss et al. (2011). Their study provided the rotation periods of 33 out of 38 members.

In the light of these studies, we also explored the most recent literature and, as a result, we finally compiled a new list of
117 stars among members and candidate members with spectral types later than about F3V. Based on this, we started a new rotational study on this enlarged sample. To get the photometric rotation periods of our targets, we used our own observations, archive data, and also made use of periods from the literature.

As result of our photometric investigation, we obtained the rotation periods of 112 out of 117 stars. Specifically, for the first time we measured the rotation periods of 51 stars. For 28 other stars, with our analysis we were able to confirm the values of new or archived data reported in the literature. For 33 stars we adopted the literature values. For the remaining five stars, our periodogram analysis did not provide the rotation period. The results of this investigation are presented in the catalogue of photometric rotation periods of the $\beta$ Pictoris association members (Messina et al. 2016, Paper I) where we describe the photometric observations that were newly obtained, 


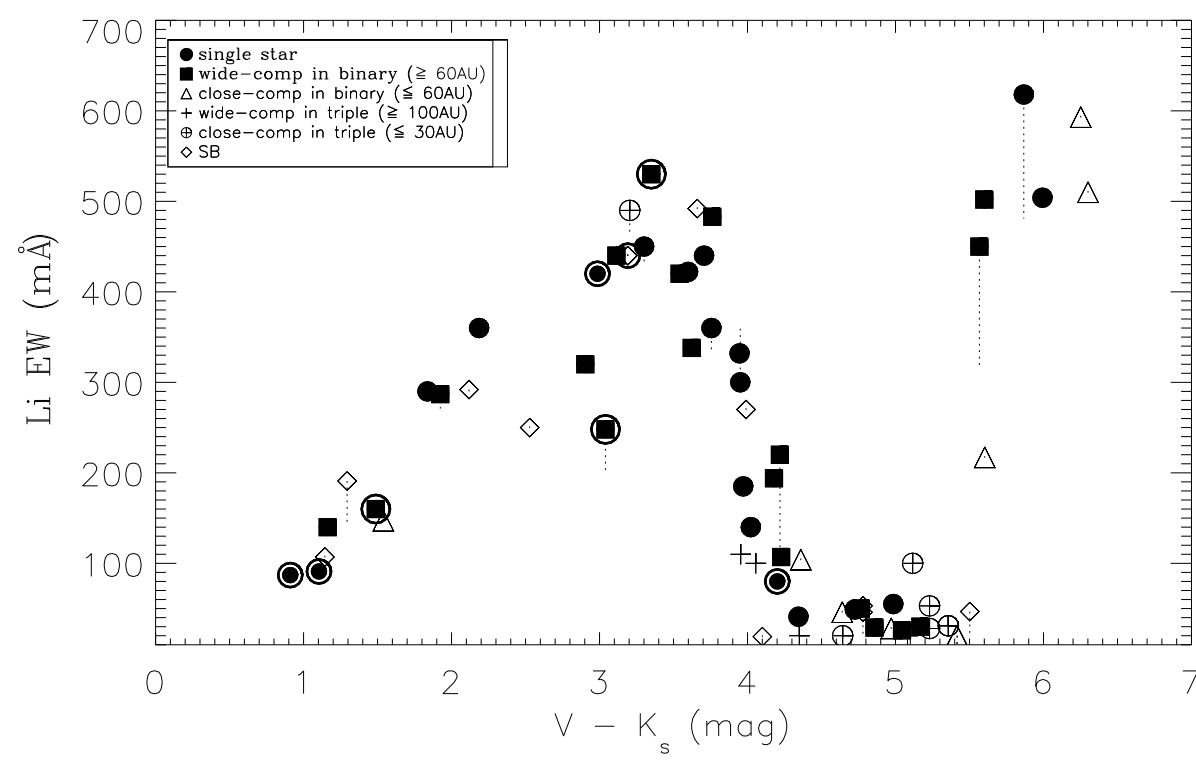

Fig. 1. Distribution of Li EW versus $V-K_{\mathrm{s}}$ color for 66 members of the $\beta$ Pictoris association. The meaning of symbols is given in the legend. Dotted vertical lines indicate the range of values for stars with multiple Li EW measurements. All circled symbols indicate stars hosting debris discs. their reduction and analysis, and a detailed discussion of our results obtained for each individual star.

In this paper (Paper II), we focus on a sub-sample consisting of 66 members for which we know the rotation period and have at least one measurement (from the literature) of the lithium equivalent width (EW). To date, this sub-sample is the largest of any known young loose association. We use it to investigate the correlation between rotation and $\mathrm{Li}$ depletion and to compare with earlier results the age of the $\beta$ Pictoris association obtained by the modeling of the lithium depletion boundary (LDB), after decorrelating the Li EW from rotation.

\section{Sample description}

The sample under analysis consists of 66 members, with a spectral type later than F3V with one measurement, at least, of the Li EW (see Table A.1). This sample, which has significantly increased with respect to earlier studies, makes a new investigation on the age of the $\beta$ Pictoris association necessary with the LDB modeling method. In fact, earlier studies made use of a smaller number of association members. Mentuch et al. (2008) made use of a sample of 23 members; Macdonald \& Mullan (2010) used a sample of only 10 members. Binks \& Jeffries (2014) and Malo et al. (2014b) used about 40 and 34 members, respectively.

As a result, we now know the rotation periods of all 66 stars, which enables us to get more accurate results and to explore the mechanism of Li depletion deeply. More specifically, for the first time we measured the rotation periods of 30 out of 66 members. These new measurements are presented in the mentioned catalogue containing the photometric rotation periods of all 112 members/candidate members of the association. For another 16 members, the rotation periods were taken from Messina et al. (2010, 2011). The remaining 20 rotation periods were taken from different literature sources.

To date, this sample represents one of the three largest samples ever used to investigate the correlation between rotation period and Li depletion. The other samples consist of members of the Pleiades and of the M34 stellar open clusters investigated by Gondoin (2014) using the rotation periods from Hartman et al. (2010), and of members of the NGC 2264 open cluster whose results on the lithium-rotation connection are presented by Bouvier et al. (2016). The earlier larger sample of
Pleiades members analysed by Soderblom et al. (1993) made use of the projected rotational velocity $(v \sin i)$, which is less accurate owing to the uncertainty arising from the unknown $\sin i$.

\section{Li distribution}

In Fig. 1, we plot the distribution of Li EW for all 66 members versus the $V-K_{\mathrm{s}}$ color. This color index is measured using the $K_{\mathrm{s}}$ magnitude from the 2MASS project (Cutri et al. 2003) and the brightest (presumably unspotted) $V$ magnitude from the long photometric time series available in the All Sky Automated Survey (ASAS) archive (Pojmanski 2002). This choice enables us to reduce the impact of photometric variability on the $V$-band magnitude. Among different color indexes, the $V-K_{\mathrm{s}}$ turned out to be the most homogeneous and with the largest range of values, enabling an accurate representation of different masses. We applied no color correction for interstellar absorption since the members under study have distances in the range from $10 \mathrm{pc}$ to $80 \mathrm{pc}$, therefore the interstellar reddening can be considered negligible. The average uncertainty on this color is $\sigma_{V-K_{\mathrm{s}}}=0.03 \mathrm{mag}$. There are only eight stars that belong to close binaries that are not resolved either in $V$ or in $K_{\mathrm{s}}$ magnitudes and consist of components that differ by no more than two spectral sub-classes. For these stars the measured $V-K_{\mathrm{s}}$ for the primary component, the one with measurement of Li EW, resulted in being redder than the expected value for the spectral type. In these cases we adopted the $V-K_{\mathrm{s}}$ values from Pecaut \& Mamajek (2013), which corresponded to the spectral type of the primary component. The Li EW measurements are retrieved from Mentuch et al. (2008), da Silva et al. (2009), Kiss et al. (2011), Binks \& Jeffries (2014), Moór et al. (2013), Malo et al. (2014b), Reid et al. (2002), Torres et al. (2006), and Desidera et al. (2015). The average uncertainty associated with these measurements is generally not larger than $\sim 40 \mathrm{~m} \AA$. The error bars associated with the measurements (in both axes) are equal to the size of the symbols plotted in Fig. 1. In a few cases more than one measurement is available. The range of values of the Li EW in these cases is plotted with a dotted line. Almost all measurements (for a total of 20 members) in the color range $4.5<V-K_{\mathrm{s}}<5.3 \mathrm{mag}$ are upper limits. Different symbols are used to distinguish the different nature of the considered 

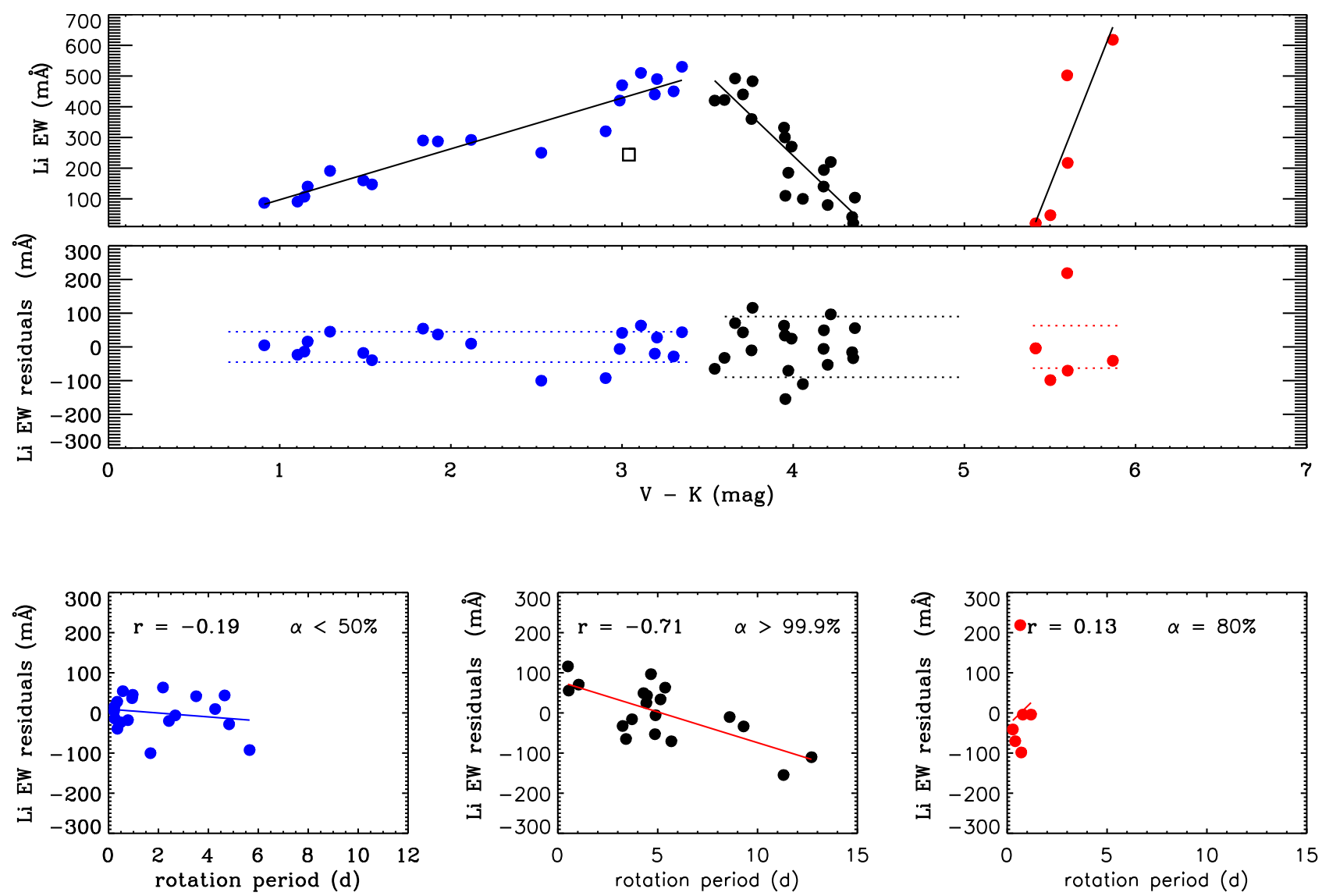

Fig. 2. Top panel: distribution of Li EW versus $V-K_{\mathrm{s}}$ color in three color ranges: $0.5<V-K_{\mathrm{s}}<3.4 \mathrm{mag}(19 \mathrm{stars}), 3.4<V-K_{\mathrm{s}}<4.5 \mathrm{mag}$ (21 stars), and $5.4<V-K_{\mathrm{s}}<5.9 \mathrm{mag}$ (6 stars) of $\beta$ Pictoris members with known rotation period and with overplotted (solid lines) the linear fits. Middle panel: residuals from the fit with horizontal dotted lines indicating the amplitude of dispersion. Bottom panels: distribution of Li EW residuals versus rotation period in the three color ranges. Solid lines are linear fits, $r$ is the Pearson linear correlation coefficient and $\alpha$ the significance level.

members (see the legend in Fig. 1). Detailed information for each member is given in Paper I and the criterion to distinguish close from wide components is given in Messina et al. (Paper III, in prep.).

In Fig. 1, we note three important features. First, we note a very well defined LDB that will be used for an estimate of the age. Second, in the color range $3.2<V-K_{\mathrm{s}}<4.5 \mathrm{mag}$, we note, even by just a visual inspection, a scatter in the Li EW that is significantly larger than in the range of bluer members. We investigate the role played by the rotation in producing this scattered depletion. Finally, we note that in the range $2.5<V-K_{\mathrm{s}}<$ $3.0 \mathrm{mag}$, we only have three stars that all have Li EWs significantly lower than the average. This color range corresponds to $\mathrm{K} 2-\mathrm{K} 3$ spectral types and is reminiscent of the Li dip observed in more evolved main-sequence stars (see, e.g., Balachandran 1995). One of these stars is HIP 84586, a SB2 spectroscopic binary whose tidal locking between the two components may have altered the internal mixing and, consequently, enhanced the $\mathrm{Li}$ depletion rate with respect to single stars. The two other stars are TYC 687801951 and HIP 11437A, both components of wide binaries that are expected to have evolved, rotationally, as single stars. We only note that HIP 11437A hosts a debris disc.

\section{The rotation-Li depletion correlation}

We first analyse the possible correlation between rotation and Li EW to investigate what role the rotation plays in producing the observed Li EW dispersion among stars with similar masses.
This information will be used for a more accurate modeling of the LBD to infer the association age.

We analyse the following three sub-groups separately: blue stars with $0.5 \leq V-K_{\mathrm{s}}<3.4$ mag totaling 19 members, red stars with $3.4 \leq V-K_{\mathrm{s}} \leq 4.5 \mathrm{mag}$ totaling 21 members, and very red stars with $5.4<V-K_{\mathrm{s}}<5.9 \mathrm{mag}$ totaling 6 members that correspond to the onset of the Li depletion. Stars with $V-K_{\mathrm{s}}>5.9 \mathrm{mag}$ have not undergone any Li depletion. In the blue stars sample, we observe a trend of increasing $\mathrm{Li} \mathrm{EW}$ with increasing color (decreasing mass); in the red stars sample, we observe the opposite trend; in the very red star sample, we again observe a trend of increasing Li EW with increasing color. As shown in the top panel of Fig. 2, linear fits are enough for our purposes, i.e., approximating the mass dependence to outline the correlation with the rotation period. In the middle panel, we plot the residuals with respect to the linear fits. Only one star (HIP 11437A), represented by a square and significantly deviating from the trend, was excluded from the fit. Positive residuals indicate stars that are more $\mathrm{Li}$ rich than the average, whereas negative residuals indicate stars that are more Li-depleted than the average. In the case of blue stars, we find a peak-to-peak amplitude of the dispersion $\sigma=90 \mathrm{~m} \AA$ of the residuals from the fit, whereas in the case of red stars the peak-to-peak amplitude of the dispersion is about twice as large, $\sigma=180 \mathrm{~m} \AA$. Finally, in the case of very red stars, we find $\sigma=125 \mathrm{~m} \AA$. The dotted lines represent peak-to-peak amplitudes of dispersions in the three color ranges. Now, to probe any dependence of the residual dispersion on the rotation period, we computed the Pearson linear 

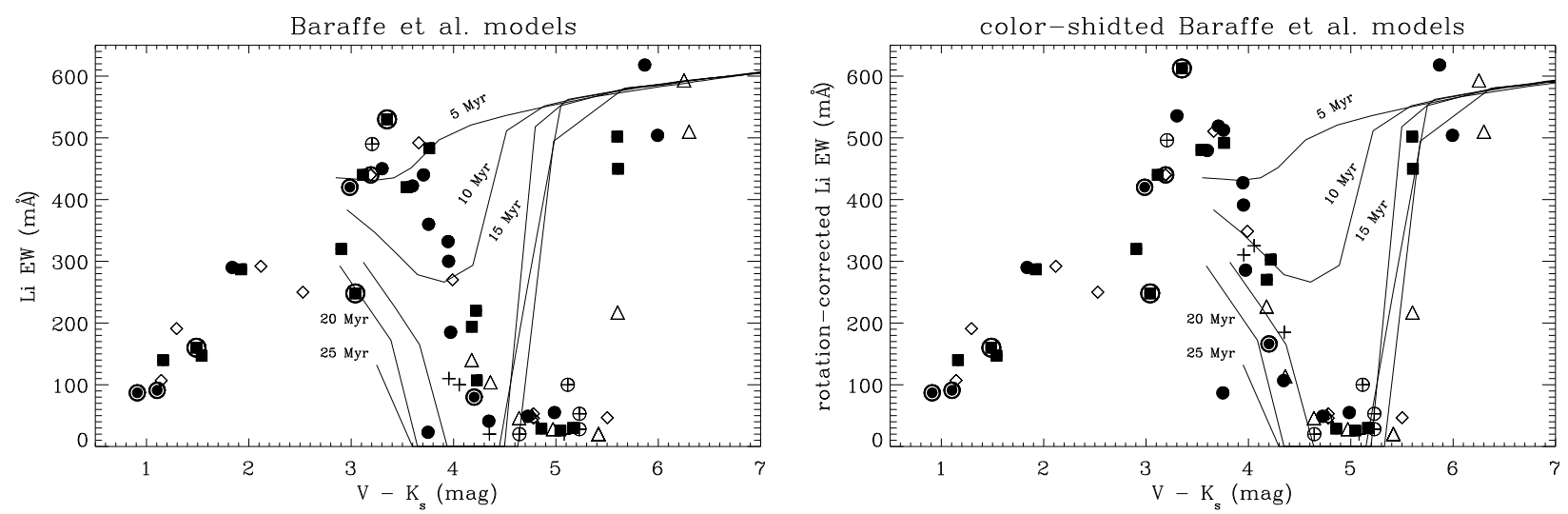

Fig. 3. Left panel: distribution of Li EWs versus $V-K_{\mathrm{s}}$ color for $\beta$ Pic members. Solid lines represent the Li EWs predicted by the models of Baraffe et al. (2015) for ages in the range from 5 to 25 Myr. Right panel: same as in the left panel, but with models arbitrarily shifted by 0.7 mag towards redder colors to match the observations.

correlation coefficients (r) and significance levels ${ }^{1}(\alpha)$ between the residuals and the rotation period, as well as the Spearman's rank correlation (Spearman 1904). In the case of blue stars we find $r=-0.19$ with a significance level $\alpha<50 \%$, i.e., no dependence exists of the residuals on rotation (from Spearman's rank correlation we found $\rho=-0.04$ with very small significance $p$ value $=0.77$ ). On the contrary, for red stars we found $r=-0.71$ with very high significance level $\alpha>99.9 \%$ (similarly, from rank correlation we found $\rho=-0.56$ with high significance $p$ value $=0.01$ ). Finally, for very red stars, we find $r=+0.13$ with a significance level $\alpha \sim 80 \%$ (from Spearman's rank correlation we found $\rho=0.29$ with very small significance $p$-value $=0.58$ ). Consequently, in this regime of very red stars, where we do not have enough data, we cannot asses the existence or not of a rotation-Li depletion connection.

In the case of red stars $\left(3.2 \leq V-K_{\mathrm{s}} \leq 4.5 \mathrm{mag}\right)$ we can state that the $\mathrm{Li}$ depletion is significantly correlated to rotation that seems to maintain fast rotators less depleted than slow rotators according to Eq. (1):

$\delta E W(\mathrm{Li})=101 \pm 22-17.7 \pm 3.4 \times P$,

where the Li EW is in $\mathrm{m} \AA$ and the rotation period $P$ in days. The average uncertainty on the rotation period $(\Delta P=0.7 \%)$ is negligible with respect to that of the Li EW. After decorrelating the Li EW from the rotation of red stars, the dispersion turns out to be reduced by about a factor of $2, \sigma=50 \mathrm{~m} \AA$ and becomes comparable with that measured among blue stars, where no dependence on rotation was observed, and among very red stars. This residual dispersion is larger than the uncertainty associated with the Li EW measurements. Therefore, apart from mass and rotation, other causes must play a role in producing the observed dispersion. The intrinsic variability of the Li EW is certainly one cause. In fact, we know that spotted stars, as are all our targets, exhibit a rotational modulation of the Li EW. Specifically, the EW is larger at the rotation phases when the spots are most clearly in view, and, consequently, the average surface temperature is lower. In our sample, 26 stars have two EW measurements. We find that the average difference between these multiple measurements is $\langle\Delta E W\rangle=26 \mathrm{m \AA}$. We note only two extreme cases: TX Psa and 2MASS J05082729-2101444, both very red stars with $\triangle E W>100 \mathrm{~m} \AA$.

1 The significance level $\alpha$ represents the probability of observing a value of the correlation coefficient larger than $r$ for a random sample having the same number of observations and degrees of freedom (Bevington 1969).
Generally, the rotational Li variability in young stars is not larger than about 5\% (see Appendix in Bouvier et al. 2016). In our case, this corresponds to a range from $\sim 5$ to $25 \mathrm{~m} \AA$ in absolute values, which is of the same order of the intrinsic variability we found.

\subsection{Observed versus predicted LBD}

It is interesting to make a comparison between the measured distribution of Li EWs and the predictions of evolutionary models. In the following, we first consider the evolutionary models of Baraffe et al. (2015) for solar abundances. Solar abundances have been measured in a few members, like $\beta$ Pic and PZ Tel, and generally they were adopted for the whole association (see, e.g., Mentuch et al. 2008). The surface Li abundance is first derived from the ratio of surface $\mathrm{Li}$ abundance to initial abundance provided with the models, using an initial abundance of $\mathrm{Li}_{0}=$ $1 \times 10^{-9}$. Then, to make the comparison with the observations, we transformed the model Li abundance into Li EW. For this purpose, we used the curves of growth from Zapatero Osorio et al. (2002). These are valid in the effective temperature range $2600<T_{\text {eff }}<4100 \mathrm{~K}$ and for $1.0<A(\mathrm{Li})<3.4$.

In the left panel of Fig. 3, we plot the measured Li EW and compare this distribution with the Li EW derived from Baraffe et al. (2015) models. We note a strong mismatch between observations and model predictions. For ages in the 10-25 Myr range, the models predict a substantial/complete depletion in the $V-K_{\mathrm{s}}$ color range 3.8-4.5 mag, whereas the Li gap is observed at colors that are redder by about $0.7 \mathrm{mag}$. In fact, a better match is achieved if we arbitrarily shift the model colors by $\Delta\left(V-K_{\mathrm{s}}\right)=+0.7 \mathrm{mag}$ (see right panel of Fig. 3 ). Moreover, assuming that the fast rotators have rotation-unaffected $\mathrm{Li}$ abundances, we maintained their observed Li EW, whereas for the slower rotators we have increased the Li EW according to Eq. (1). As shown in the right panel of Fig. 3, we find that, even applying this $V-K_{\mathrm{s}}$ color shift and the rotation decorrelation, we can infer different ages by looking at different parts of the observed Li depletion since the slope of the observed depletion is different to that predicted by the models.

We note that the same mismatch between observations and models is found when different colors (e.g., $V-I, J-H$, or $J-K$ ) or the effective temperatures are used to make the comparison. The colors of the stars in the Li depletion gap $4.4<$ $V-K_{\mathrm{s}}<5.4 \mathrm{mag}$ have been accurately checked. For a few 


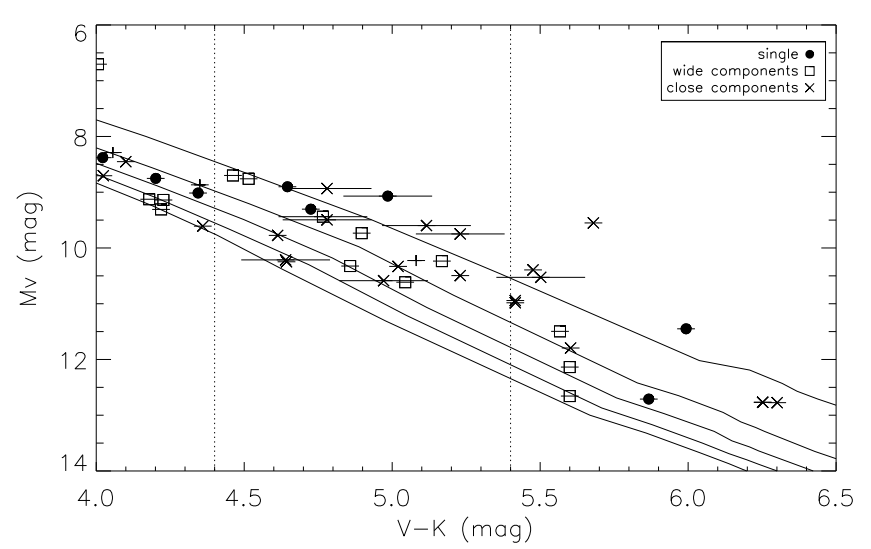

Fig. 4. Absolute magnitude versus $V-K_{\mathrm{s}}$ color of members in the $\mathrm{Li}$ gap. Solid lines represent the isochrones corresponding to ages from 5 (top) to $25 \mathrm{Myr}$ (bottom).

stars (either single or resolved components in wide binaries) the $V-K_{\mathrm{s}}$ is measured for each component. For other stars (components of unresolved binaries) the $V-K_{\mathrm{s}}$ is derived from the spectral type of the primary component, which is the one with measured Li. In the first case, the error on $V-K_{\mathrm{s}}$ is $\pm 0.03 \mathrm{mag}$, in the latter case is \pm 0.15 mag. In Fig. 4 we compare the absolute magnitudes of the members in the $\mathrm{Li}$ gap with isochrones for ages from 5 to $25 \mathrm{Myr}$ taken from Baraffe et al. (2015). We note that within the gap $\left(4.4<V-K_{\mathrm{s}}<5.4 \mathrm{mag}\right)$ the stars seem to be either more luminous or redder than expected for the most recent age estimation of $24 \mathrm{Myr}$ by Bell et al. (2015). A better agreement is observed at bluer colors, where stars are within the 10-25 Myr isochrones. If we correct the magnitudes of close components for binarity, we get a better agreement, but do not solve the overluminosity. This indicates that, in order to reproduce the data, the predicted Baraffe et al. (2015) colours should be shifted redward.

All stars in the gap have a prominent magnetic activity as is inferred from photometric variability, X-ray emission, and near-UV/far-UV excess. Therefore, models including the effect of magnetic fields should be more adequate for describing the Li depletion pattern. For this reason, we also made use of the Dartmouth stellar evolutionary models that incorporate the effects of magnetic fields (Feiden 2016; Mann et al. 2016). In these models, inhibition of convection by magnetic fields cools the stellar surface temperature, thereby slowing the contraction rate of young stars. Stars have a larger radius and a higher luminosity at a given age, as a result

These models were computed for solar metallicity and for an equipartition magnetic field strength in the range $2500<$ $B f_{\text {eq }}<3000 \mathrm{G}$. Model effective temperatures were transformed into $V-K_{\mathrm{s}}$ colors using the empirical $T_{\text {eff }}-\left(V-K_{\mathrm{s}}\right)$ relation from Pecaut \& Mamajek (2013) valid for young 5-30 Myr stars. $\mathrm{Li}$ abundances were transformed into $\mathrm{Li} \mathrm{EW}$, as performed in the case of the Baraffe et al. models.

In the left panel of Fig. 5, we plot the measured Li EW and compare this distribution with the Li EW derived from the Dartmouth et al. models. In this case, no arbitrary color shift is needed to match the hot side of the Li gap. A better match is achieved in the right panel of Fig. 5, when the Li EW are decorrelated from rotation, assuming again that the fast rotators have rotation-unaffected $\mathrm{Li}$ abundances.
The reduced chi-squares ${ }^{2}$ computed from the fit to the hot boundary of the Li gap have their minimum (see Fig. 6) for an age of $25 \pm 3 \mathrm{Myr}$. The uncertainty formally represents the age interval by which the $\chi_{v}^{2}$ increases by one unity from the minimum value. The relatively large values of the $\chi_{v}^{2}$ indicate either that the EW scatter arising from the intrinsic variability (rotational modulation) is larger than our estimate of $26 \mathrm{~m} \AA$, or the uncertainties associated with the measurements are, in several cases, underestimated at some level.

Our age estimation is in good agreement with respect to the more recent age estimates of $23 \pm 4 \mathrm{Myr}$ and $24 \pm 3 \mathrm{Myr}$ by Mamajek \& Bell (2014) and Bell et al. (2015), respectively, and significantly lower than the Macdonald \& Mullan (2010) estimate.

\section{Conclusions}

We have retrieved from the literature the measured Li EW of 66 members of the young $\beta$ Pictoris association. We have carried out a photometric monitoring of these members that allowed us to measure, for the first time, the rotation periods of $30 \mathrm{mem}$ bers. For 16 other members we retrieved the rotation periods from Messina et al. $(2010,2011)$ and, for the remaining members, we used different sources in the literature.

We have explored the existence of a connection between rotation and Li depletion. After removing the mass dependence of the $\mathrm{Li} \mathrm{EW}$, using linear fits to $\mathrm{EW}$ versus $V-K_{\mathrm{s}}$, we found that for stars with $0.5 \leq V-K_{\mathrm{s}} \leq 3.4 \mathrm{mag}$, which roughly corresponds to masses $M>0.8 M_{\odot}$, no significant correlation is found between Li EW and rotation period. On the contrary, in the color range $3.4 \leq V-K_{\mathrm{s}} \leq 4.5 \mathrm{mag}$, which roughly corresponds to masses $0.3<M<0.8 M_{\odot}$, we find a strong correlation between the Li EW and the rotation period, where fast rotators are much less Li-depleted than slow rotators. Finally, in the color range $5.4<V-K_{\mathrm{s}}<5.9 \mathrm{mag}$, roughly corresponding to masses $M \sim 0.1 M_{\odot}$, we have some hint of an inverted correlation, where fast rotators are more depleted than slow rotators. However, this correlation is currently based on only seven stars and the significance level is high $(80 \%)$, but this is due to just one point that could be an outlier for whatever reason.

Interestingly, the dispersion in the $3.4 \leq V-K_{\mathrm{s}} \leq 4.5 \mathrm{mag}$ has a peak-to-peak amplitude that amounts to $180 \mathrm{~m} \AA$, which is about a factor of two larger than that measured in the same mass range in the 5-Myr NGC 2264 open cluster (Bouvier et al. 2016), and about a factor two smaller than that measured in the 125-Myr Pleiades open cluster (Soderblom et al. 1993). Therefore, we note that the effect of rotation on the Li depletion is also age dependent and increases with age.

A comparison with the Baraffe et al. (2015) models, shows a mismatch of about $0.7 \mathrm{mag}$ in the $V-K_{\mathrm{s}}$ color between the observed and the predicted color range where the Li gap falls. Models predict the Li gap at bluer colors than observed. On the contrary, the Dartmouth models that incorporate the effects of magnetic fields provide a good match with the hot side of the Li depletion gap, although some mismatch on the cool side of the Li gap remains. Comparing the Dartmouth models with the hot side of the Li depletion gap, we infer an age of $25 \pm 3 \mathrm{Myr}$, which is in very good agreement with the most recent age estimates for the $\beta$ Pictoris association. However, the relatively large values of the reduced chi-squares suggest that either the intrinsic Li EW

2 To compute the $\chi_{v}^{2}$ we used the uncertainty $\sigma=\sqrt{\sigma_{0}^{2}+\sigma_{v}^{2}}$ where $\sigma_{0}$ is the measurement error and $\sigma_{v}$ is the average intrinsic variability. 

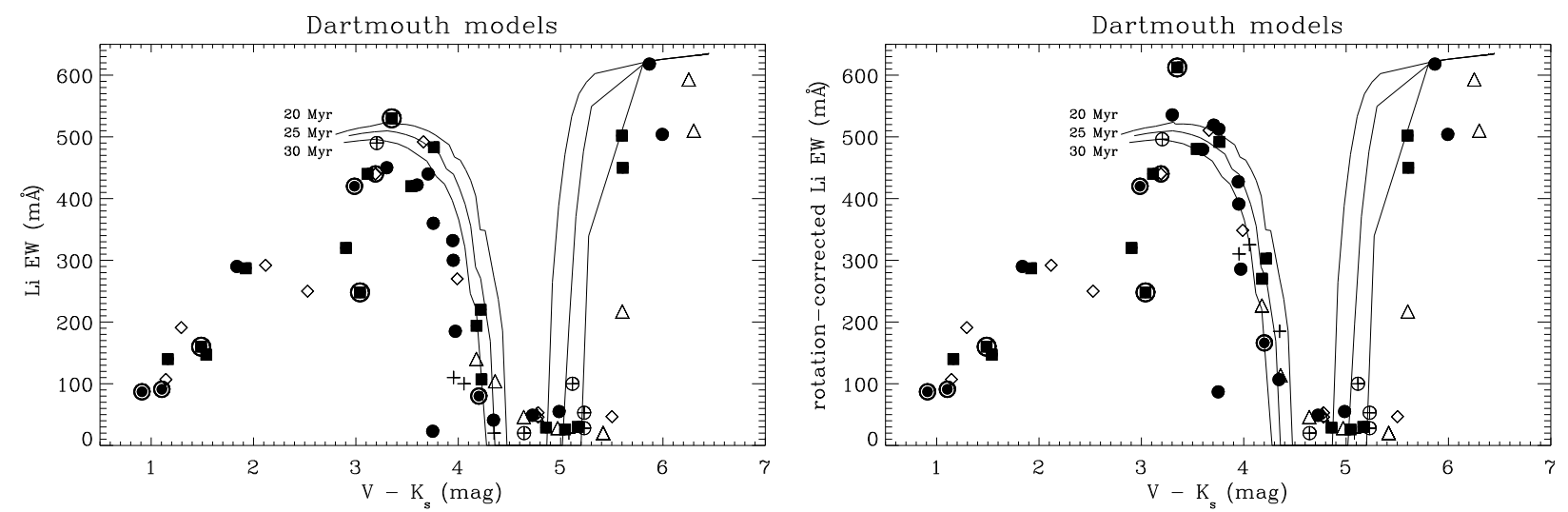

Fig. 5. Left panel: distribution of Li EWs versus $V-K_{\mathrm{s}}$ color for $\beta$ Pic members. Solid lines represent the Li EWs predicted by the Dartmouth models for ages in the range from 20 to $30 \mathrm{Myr}$. Right panel: same as in the left panel, but Li EW decorrelated from rotation, assuming that fast rotators have rotation-unaffected $\mathrm{Li} \mathrm{EW}$.

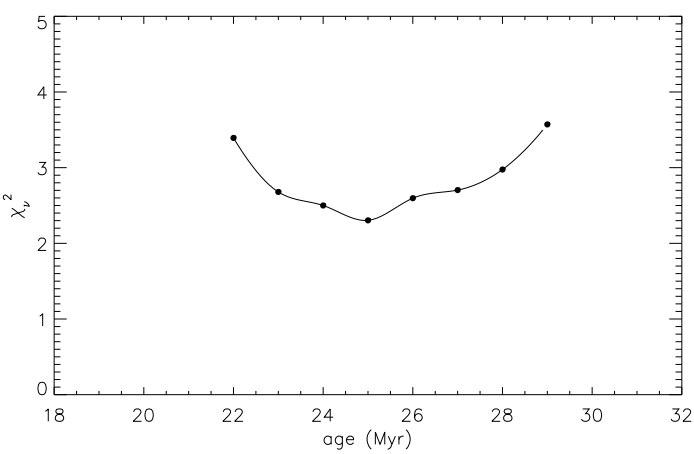

Fig. 6. Reduced chi-squares versus age obtained from the residuals from the fit to the hot boundary of the Li-depletion gap.

variability is still underestimated or some other factor, apart from rotation, plays a relevant role in producing the observed scatter among stars with similar mass.

Acknowledgements. Research on stellar activity at INAF- Catania Astrophysica Observatory is supported by MIUR (Ministero dell'Istruzione, dell'Universitá e della Ricerca). This research has made use of the Simbad database, operated at CDS (Strasbourg, France). S.M. thanks Jerome Bouvier for useful discussion and the anonymous referee for useful comments that helped us to improve this paper.

\section{References}

Balachandran, S. 1995, in The Light Element Abundances (Springer) Baraffe, I., Homeier, D., Allard, F., \& Chabrier, G. 2015, A\&A, 577, A42 Bell, C. P. M., Mamajek, E. E., \& Naylor, T. 2015, MNRAS, 454, 593

Bevington, P. R. 1969, Data reduction and error analysis for the physical sciences (New York: McGraw-Hill)

Binks, A. S., \& Jeffries, R. D. 2014, MNRAS, 438, L11
Bouvier, J., Lanzafame, A. C., Venuti, L., et al. 2016, A\&A, 590, A78 Cutri, R. M., Skrutskie, M. F., van Dyk, S., et al. 2003, 2MASS All Sky Catalog of point sources (NASA/IPAC)

da Silva, L., Torres, C. A. O., de La Reza, R., et al. 2009, A\&A, 508, 833

Desidera, S., Covino, E., Messina, S., et al. 2015, A\&A, 573, A126

Feiden, G. A. 2016, A\&A, 593, A99

Gondoin, P. 2014, A\&A, 566, A72

Hartman, J. D., Bakos, G. Á., Kovács, G., \& Noyes, R. W. 2010, MNRAS, 408, 475

Kiss, L. L., Moór, A., Szalai, T., et al. 2011, MNRAS, 411, 117

Lépine, S., \& Simon, M. 2009, AJ, 137, 3632

Macdonald, J., \& Mullan, D. J. 2010, ApJ, 723, 1599

Malo, L., Doyon, R., Lafrenière, D., et al. 2013, ApJ, 762, 88

Malo, L., Artigau, É., Doyon, R., et al. 2014a, ApJ, 788, 81

Malo, L., Doyon, R., Feiden, G. A., et al. 2014b, ApJ, 792, 37

Mamajek, E. E., \& Bell, C. P. M. 2014, MNRAS, 445, 2169

Mann, A. W., Newton, E. R., Rizzuto, A. C., et al. 2016, AJ, 152, 61

Mentuch, E., Brandeker, A., van Kerkwijk, M. H., Jayawardhana, R., \& Hauschildt, P. H. 2008, ApJ, 689, 1127

Messina, S., Desidera, S., Turatto, M., Lanzafame, A. C., \& Guinan, E. F. 2010, A\&A, 520, A15

Messina, S., Desidera, S., Lanzafame, A. C., Turatto, M., \& Guinan, E. F. 2011, A\&A, 532, A10

Messina, S., Millward, M., Buccino, A., et al. 2016, A\&A, in press (Paper I) DOI: $10.1051 / 0004-6361 / 201629152$

Moór, A., Szabó, G. M., Kiss, L. L., et al. 2013, MNRAS, 435, 1376

Pecaut, M. J., \& Mamajek, E. E. 2013, ApJS, 208, 9

Pojmanski, G. 2002, Acta Astron., 52, 397

Reid, I. N., Kirkpatrick, J. D., Liebert, J., et al. 2002, AJ, 124, 519

Schlieder, J. E., Lépine, S., \& Simon, M. 2010, AJ, 140, 119

Schlieder, J. E., Lépine, S., \& Simon, M. 2012, AJ, 143, 80

Shkolnik, E. L., Anglada-Escudé, G., Liu, M. C., et al. 2012, ApJ, 758, 56

Soderblom, D. R., Jones, B. F., Balachandran, S., et al. 1993, AJ, 106, 1059

Spearman, C. 1904, Am. J. Psychol., 15, 72

Torres, C. A. O., Quast, G. R., da Silva, L., et al. 2006, A\&A, 460, 695

Zapatero Osorio, M. R., Béjar, V. J. S., Pavlenko, Y., et al. 2002, A\&A, 384, 937

Zuckerman, B., \& Song, I. 2004, ARA\&A, 42, 685 
S. Messina et al.: Rotation - lithium correlation in the $\beta$ Pic association

\section{Appendix A: Additional table}

Table A.1. Properties of the 66 members of the $\beta$ Pictoris association studied in this work.

\begin{tabular}{|c|c|c|c|c|c|c|c|c|c|c|c|}
\hline \multirow{2}{*}{$\begin{array}{l}\text { Target } \\
\text { HIP 560 }\end{array}$} & \multicolumn{3}{|c|}{$\begin{array}{c}\mathrm{RA} \\
(\mathrm{hh}, \mathrm{mm}, \mathrm{ss})\end{array}$} & \multicolumn{3}{|c|}{$\begin{array}{c}\text { Dec } \\
\left({ }^{\circ},{ }^{\prime},{ }^{\prime \prime}\right)\end{array}$} & \multirow{2}{*}{$\begin{array}{l}\text { Sp.T } \\
\text { F3V }\end{array}$} & \multirow{2}{*}{$\begin{aligned} \begin{array}{l}V \\
(\mathrm{mag})\end{array} \\
6.15\end{aligned}$} & \multirow{2}{*}{$\begin{array}{r}V-K_{\mathrm{s}} \\
(\mathrm{mag})\end{array}$} & \multirow{2}{*}{$\begin{array}{c}P \\
\text { (d) } \\
0.224\end{array}$} & \multirow{2}{*}{$\begin{array}{r}\mathrm{Li} E W \\
(\mathrm{~mA})\end{array}$} \\
\hline & 00 & 06 & 50.08 & -23 & 06 & 27.20 & & & & & \\
\hline TYC 1186-0706-1 & 00 & 23 & 34.66 & +20 & 14 & 28.75 & $\mathrm{~K} 7.5 \mathrm{~V}+\mathrm{M} 5$ & 10.96 & 3.623 & 7.900 & 338.0 \\
\hline GJ 2006A & 00 & 27 & 50.23 & -32 & 33 & 06.42 & $\mathrm{M} 3.5 \mathrm{Ve}$ & 12.87 & 4.858 & 3.990 & 29.0 \\
\hline GJ 2006B & 00 & 27 & 50.35 & -32 & 33 & 23.86 & $\mathrm{M} 3.5 \mathrm{Ve}$ & 13.16 & 5.044 & 4.910 & 26.0 \\
\hline 2MASS J01112542+1526214A & 01 & 11 & 25.42 & +15 & 26 & 21.50 & M5+M6 & 14.46 & 6.252 & 0.911 & 593.0 \\
\hline 2MASS J01351393-0712517 & 01 & 35 & 13.93 & -07 & 12 & 51.77 & $\mathrm{M} 4.5 \mathrm{~V}$ & 13.22 & 5.502 & 0.703 & 46.7 \\
\hline TYC 1208-0468-1 & 01 & 37 & 39.42 & +18 & 35 & 32.91 & $\mathrm{~K} 3+\mathrm{K} 5$ & 9.85 & 3.114 & 2.803 & 440.0 \\
\hline HIP 10679 & 02 & 17 & 24.74 & +28 & 44 & 30.43 & $\mathrm{G} 2 \mathrm{~V}$ & 7.75 & 1.488 & 0.777 & 160.0 \\
\hline HIP 10680 & 02 & 17 & 25.28 & +28 & 44 & 42.16 & F5V & 6.95 & 1.163 & 0.240 & 140.0 \\
\hline HIP 11437A & 02 & 27 & 29.25 & +30 & 58 & 24.60 & $\mathrm{~K} 4$ & 10.12 & 3.040 & 12.500 & 248.0 \\
\hline HIP 11437B & 02 & 27 & 28.05 & +30 & 58 & 40.53 & M1 & 12.44 & 4.219 & 4.660 & 220.0 \\
\hline HIP 12545 & 02 & 41 & 25.90 & +05 & 59 & 18.00 & K6Ve & 10.37 & 3.301 & 4.830 & 450.0 \\
\hline GJ3305 & 04 & 37 & 37.30 & -02 & 29 & 28.00 & $\mathrm{M} 1+\mathrm{M} ?$ & 10.59 & 4.177 & 4.890 & 140.0 \\
\hline 2MASS J04435686+3723033 & 04 & 43 & 56.87 & +37 & 23 & 03.30 & M3Ve+M5? & 12.98 & 4.179 & 4.288 & 194.0 \\
\hline HIP 23200 & 04 & 59 & 34.83 & +01 & 47 & 00.68 & $\mathrm{M} 0.5 \mathrm{Ve}$ & 10.05 & 3.990 & 4.430 & 270.0 \\
\hline HIP 23309 & 05 & 00 & 47.10 & -57 & 15 & 25.00 & MoVe & 10.00 & 3.756 & 8.600 & 360.0 \\
\hline HIP 23418A & 05 & 01 & 58.80 & +09 & 59 & 00.00 & M3V & 11.45 & 4.780 & 1.220 & 53.0 \\
\hline BD - $211074 \mathrm{~A}$ & 05 & 06 & 49.90 & -21 & 35 & 09.00 & $\mathrm{M} 1.5 \mathrm{~V}$ & 10.29 & 4.350 & 9.300 & 20.0 \\
\hline BD -211074B & 05 & 06 & 49.90 & -21 & 35 & 09.00 & $\mathrm{M} 2.5 \mathrm{~V}$ & 11.67 & 4.643 & 5.400 & 20.0 \\
\hline 2MASS J05082729-2101444 & 05 & 08 & 27.30 & -21 & 01 & 44.40 & M5.6V & 14.70 & 5.867 & 0.280 & 618.0 \\
\hline 2MASS J05241914-1601153 & 05 & 24 & 19.15 & -16 & 01 & 15.30 & M4.5+M5 & 13.50 & 5.603 & 0.401 & 217.0 \\
\hline HIP 25486 & 05 & 27 & 04.76 & -11 & 54 & 03.47 & F7V & 6.22 & 1.294 & 0.966 & 191.0 \\
\hline 2MASS J05335981-0221325 & 05 & 33 & 59.81 & -02 & 21 & 32.50 & $\mathrm{M} 2.9 \mathrm{~V}$ & 12.42 & 4.725 & 7.250 & 49.0 \\
\hline 2MASS J06131330-2742054 & 06 & 13 & 13.31 & -27 & 42 & 05.50 & M3.V: & 12.09 & 5.230 & 16.9 & 28.0 \\
\hline HIP 29964 & 06 & 18 & 28.20 & -72 & 02 & 41.00 & $\mathrm{~K} 4 \mathrm{Ve}$ & 9.80 & 2.986 & 2.670 & 420.0 \\
\hline TWA 22 & 10 & 17 & 26.89 & -53 & 54 & 26.50 & M5 & 13.99 & 6.301 & 0.830 & 510.0 \\
\hline HIP 76629 & 15 & 38 & 57.50 & -57 & 42 & 27.00 & $\mathrm{~K} 0 \mathrm{~V}$ & 7.97 & 2.118 & 4.270 & 292.0 \\
\hline 2MASS J16430128-1754274 & 16 & 43 & 01.29 & -17 & 54 & 27.50 & M0.6 & 12.50 & 3.951 & 5.140 & 300.0 \\
\hline HIP 84586 & 17 & 17 & 25.50 & -66 & 57 & 04.00 & G5IV+K5IV & 7.23 & 2.528 & 1.680 & 250.0 \\
\hline HD $155555 \mathrm{C}$ & 17 & 17 & 31.29 & -66 & 57 & 05.49 & $\mathrm{M} 3.5 \mathrm{Ve}$ & 12.71 & 5.081 & 4.430 & 20.0 \\
\hline TYC 872822621 & 17 & 29 & 55.10 & -54 & 15 & 49.00 & K1V & 9.55 & 2.186 & 1.830 & 360.0 \\
\hline GSC 08350-01924 & 17 & 29 & 20.67 & -50 & 14 & 53.00 & M3V & 12.86 & 4.766 & 1.982 & 50.0 \\
\hline V4046 Sgr & 18 & 14 & 10.50 & -32 & 47 & 33.00 & $\mathrm{~K} 5+\mathrm{K} 7$ & 10.44 & 3.191 & 2.420 & 440.0 \\
\hline 2MASS J18151564-4927472 & 18 & 15 & 15.64 & -49 & 27 & 47.20 & $\mathrm{M} 3 \mathrm{~V}$ & 12.86 & 4.780 & 0.447 & 46.0 \\
\hline HIP 89829 & 18 & 19 & 52.20 & -29 & 16 & 33.00 & G1V & 8.89 & 1.837 & 0.571 & 290.0 \\
\hline 2MASS J18202275-1011131A & 18 & 20 & 22.74 & -10 & 11 & 13.62 & $\mathrm{~K} 5 \mathrm{Ve}+\mathrm{K} 7 \mathrm{Ve}$ & 10.63 & 3.350 & 4.650 & 530.0 \\
\hline TYC 907724891 & 18 & 45 & 37.02 & -64 & 51 & 46.14 & $\mathrm{~K} 5 \mathrm{Ve}$ & 9.30 & 3.204 & 0.345 & 490.0 \\
\hline TYC 907307621 & 18 & 46 & 52.60 & -62 & 10 & 36.00 & M1Ve & 11.80 & 3.946 & 5.370 & 332.0 \\
\hline HD 173167 & 18 & 48 & 06.36 & -62 & 13 & 47.02 & F5V & 7.28 & 1.144 & 0.250 & 107.0 \\
\hline TYC 740800541 & 18 & 50 & 44.50 & -31 & 47 & 47.00 & $\mathrm{~K} 8 \mathrm{Ve}$ & 11.20 & 3.660 & 1.075 & 492.0 \\
\hline HIP 92680 & 18 & 53 & 05.90 & -50 & 10 & 50.00 & $\mathrm{~K} 8 \mathrm{Ve}$ & 8.29 & 1.924 & 0.944 & 287.0 \\
\hline TYC 687210111 & 18 & 58 & 04.20 & -29 & 53 & 05.00 & MoVe & 11.78 & 3.762 & 0.503 & 483.0 \\
\hline 2MASS J19102820-2319486 & 19 & 10 & 28.21 & -23 & 19 & 48.60 & M4V & 13.20 & 4.985 & 3.640 & 55.0 \\
\hline TYC 687801951 & 19 & 11 & 44.70 & -26 & 04 & 09.00 & $\mathrm{~K} 4 \mathrm{Ve}$ & 10.27 & 2.904 & 5.650 & 320.0 \\
\hline 2MASS J19233820-4606316 & 19 & 23 & 38.20 & -46 & 06 & 31.60 & MOV & 11.87 & 3.598 & 3.242 & 422.0 \\
\hline TYC 744311021 & 19 & 56 & 04.37 & -32 & 07 & 37.71 & $\mathrm{M} 0.0 \mathrm{~V}$ & 11.80 & 3.954 & 11.300 & 110.0 \\
\hline 2MASS J19560294-3207186AB & 19 & 56 & 02.94 & -32 & 07 & 18.70 & M4V & 13.23 & 5.116 & 1.569 & 100.0 \\
\hline 2MASS J20013718-3313139 & 20 & 01 & 37.18 & -33 & 13 & 14.01 & M1 & 12.25 & 4.056 & 12.700 & 100.0 \\
\hline 2MASS J20055640-3216591 & 20 & 05 & 56.41 & -32 & 16 & 59.15 & M2: & 11.96 & 4.022 & 8.368 & 140.0 \\
\hline HD 191089 & 20 & 09 & 05.21 & -26 & 13 & 26.52 & F5V & 7.18 & 1.104 & 0.488 & 91.0 \\
\hline 2MASS J20100002-2801410AB & 20 & 10 & 00.03 & -28 & 01 & 41.10 & $\mathrm{M} 2.5+\mathrm{M} 3.5$ & 12.80 & 4.640 & 0.470 & 46.0 \\
\hline 2MASS J20333759-2556521 & 20 & 33 & 37.59 & -25 & 56 & 52.20 & M4.5 & 14.87 & 5.993 & 0.710 & 504.0 \\
\hline HIP 102141A & 20 & 41 & 51.20 & -32 & 26 & 07.00 & M4Ve & 10.36 & 5.416 & 1.191 & 20.0 \\
\hline HIP 102141B & 20 & 41 & 51.10 & -32 & 26 & 10.00 & M4Ve & 10.36 & 5.416 & 0.781 & 20.0 \\
\hline 2MASS J20434114-2433534 & 20 & 43 & 41.14 & -24 & 33 & 53.19 & M3.7+M4.1 & 12.73 & 4.971 & 1.610 & 28.0 \\
\hline HIP 102409 & 20 & 45 & 09.50 & -31 & 20 & 27.00 & M1Ve & 8.73 & 4.201 & 4.860 & 80.0 \\
\hline HIP 103311 & 20 & 55 & 47.67 & -17 & 06 & 51.04 & F8V & 7.35 & 1.539 & 0.356 & 147.0 \\
\hline TYC 634902001 & 20 & 56 & 02.70 & -17 & 10 & 54.00 & $\mathrm{~K} 6 \mathrm{Ve}+\mathrm{M} 2$ & 10.62 & 3.541 & 3.410 & 420.0 \\
\hline 2MASS J21100535-1919573 & 21 & 10 & 05.36 & -19 & 19 & 57.40 & $\mathrm{M} 2 \mathrm{~V}$ & 11.54 & 4.344 & 3.710 & 41.0 \\
\hline 2MASS J21103147-2710578 & 21 & 10 & 31.48 & -27 & 10 & 57.80 & $\mathrm{M} 4.5 \mathrm{~V}$ & 14.90 & 5.600 & 0.650 & 502.0 \\
\hline TYC 9486-927-1 & 21 & 25 & 27.49 & -81 & 38 & 27.68 & $\mathrm{M} 2 \mathrm{~V}$ & 11.70 & 4.360 & 0.542 & 104.0 \\
\hline TYC 221113091 & 22 & 00 & 41.59 & +27 & 15 & 13.60 & MOV & 11.39 & 3.666 & 1.109 & 40.0 \\
\hline TYC 934004371 & 22 & 42 & 48.90 & -71 & 42 & 21.00 & $\mathrm{~K} 7 \mathrm{Ve}$ & 10.60 & 3.706 & 4.460 & 440.0 \\
\hline HIP 112312 & 22 & 44 & 58.00 & -33 & 15 & 02.00 & M4Ve & 12.10 & 5.168 & 2.370 & 30.0 \\
\hline TX Psa & 22 & 45 & 00.05 & -33 & 15 & 25.80 & $\mathrm{M} 4.5 \mathrm{Ve}$ & 13.36 & 5.567 & 1.080 & 450.0 \\
\hline TYC 583206661 & 23 & 32 & 30.90 & -12 & 15 & 52.00 & MoVe & 10.54 & 3.971 & 5.680 & 185.0 \\
\hline
\end{tabular}

